\title{
Upper Limb Recovery Prediction After Stroke Rehabilitation Based On Regression Method
}

\author{
Ghada M. Bani Musa, Fady Alnajjar*, Adel Al-Jumaily and Shingo Shimoda
}

\begin{abstract}
In this paper, we investigate the possibility of a machine-learning algorithm using the Support Victor Machine Regression (SVMR) to predict the motor functional recovery of moderate post stroke patients during their rehabilitation program. To train the model, we used the recorded electromyography (EMG) signals from the upper limb muscles of the patients during their initial rehabilitation sessions. Then we tested the trained model to predict the later muscles performance of the patient during the same sessions. The results of this pilot study were promising; data were, to some extent, predictable. We believe such research direction could be essential to motivate the patient to complete the designed rehabilitation program and can assist the therapist to innovate proper rehabilitation menu for individual patients.
\end{abstract}

Keywords - Upper Limb, Rehabilitation, Regression, SVMR.

\section{INTRODUCTION}

$\mathrm{N}$ EUROLOGICAL disorders following brain stroke are classified as one of the leading cause of long-term motors disabilities worldwide. Such motor disabilities may not only affect the survivors' quality of life (QOL), but also can affect their surrounding community.

Motors disabilities after stroke do not necessarily have to be identical in terms of the location and the size of the injury, thus it is difficult for the therapist to predict the evolvement of disability, the optimal treatment, and the recovery period [1].

The muscle bio-signal, Electromyography (EMG), have been studied by many research teams to understand the development of human control systems to improve rehabilitations techniques [2][3][4]. Prediction of motor recovery after stroke, however, is still a hot topic to be addressed. In this pilot study, we used the recorded EMG data from three moderate post-stroke patients during their rehabilitation sessions to investigate the possibility to predict their upcoming motor activities.

\section{METHOD}

Three unilateral moderate post-stroke patients (age: $52+/-2$,

G. M. is PHD student in the Faculty of Eng. and Info. Technology, Uni. of Tech. Sydney, Sydney, Ghada.m.banimusa@ student.uts.edu.au.

F. A. is College of Info. Tech. (CIT), The United Arab Emirates Uni., 15551, AlAin, UAE, fady.alnajjar@uaeu.ac.ae. (*Corresponding author)

A. A. is Associate Professor with the Faculty of Eng. and Info. Technology, University of Technology Sydney, Sydney.

F.A and S. S. are with the Intelligent Behavior Control Unit, RIKEN, Japan.
SIAS score: 3) were recruited for this study. 18 EMGs data was recorded from the upper limp muscles of the patients while performing driving simulation task [5]. Nine EMGs data was recorded from the muscles on stroke-affected side, and remaining 9 EMGs data was recorded from the nonaffected side. Data were collected from two independent sessions (30 minutes were rest given between the two sessions) of approximately 60 movements each.

The initial $80 \%$ of the collected EMGs data for each session was considered as the training data set. The remaining $20 \%$ were considered as the testing data set. The training data was band-pass filtered before being processed by a regression model using the Support Victor Machine Regression (SVMR) [6]. Four different kernels were tested. We used the root mean square error (RMSE), mean square error (MSE) and mean absolute error (MAE) to check the error between the predicted data and the actual data (the testing data). The kernel with the minimum error, the fine Gaussian Kernel in this study, was used.

\section{RESULTS AND DISCUSSION}

For patient $(n=1)$, results of session 1 and 2 are as shown in Fig.1 and Fig.2, respectively. Where the brown solid circles represent the actual result and the blue solid circles represent the predicted outputs.

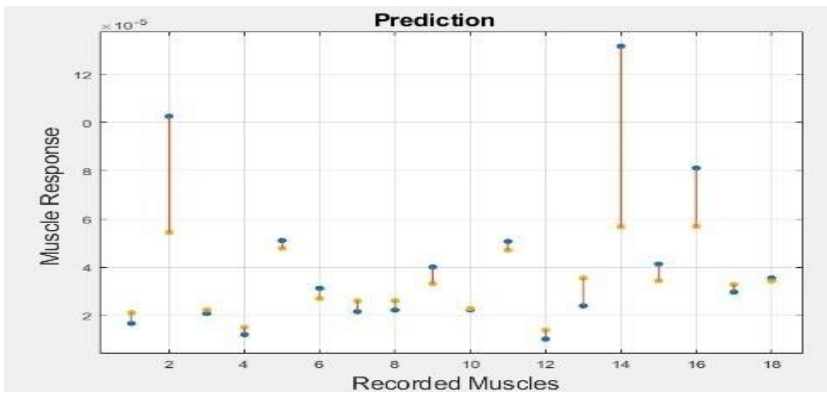

Fig. 1. Patient n1 (session 1), RMSE 3.3e-05, MSE 1.3e-09, MAE 2.3e-05.

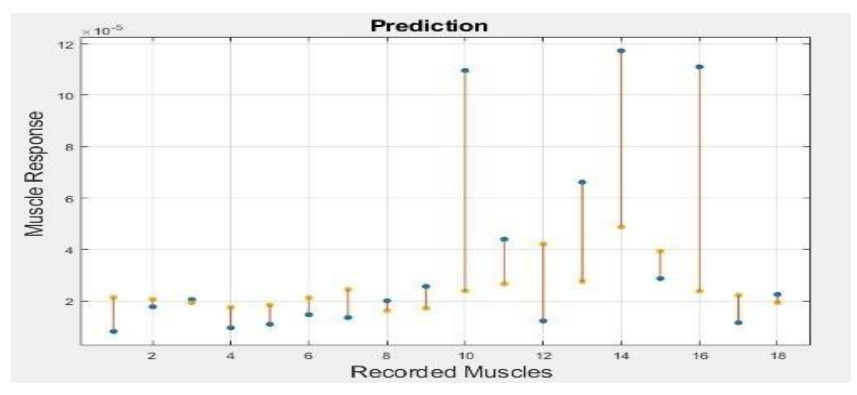

Fig. 2. Patient n1 (session 2), RMSE 2.2e-05 MSE 4.9e-10, MAE 1.1e05. 
From Fig.1\&2, the first 9 points of the $\mathrm{x}$-axis are for the stroke-affected side, while the remaining 9 points are for the non-affected side. As on the same pattern of Fig.1\&2, Fig.3 \& Fig.4/(Fig.5 \& Fig.6) represent data of patient $n=2 /(n=3)$.

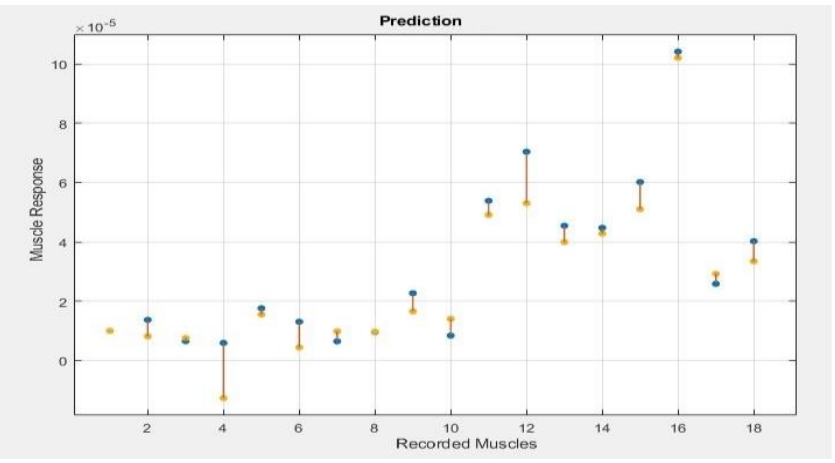

Fig. 3. Patient n2 (session 1), RMSE 2.2e-05, MSE 4.8e-10, MAE 1.6e-05.

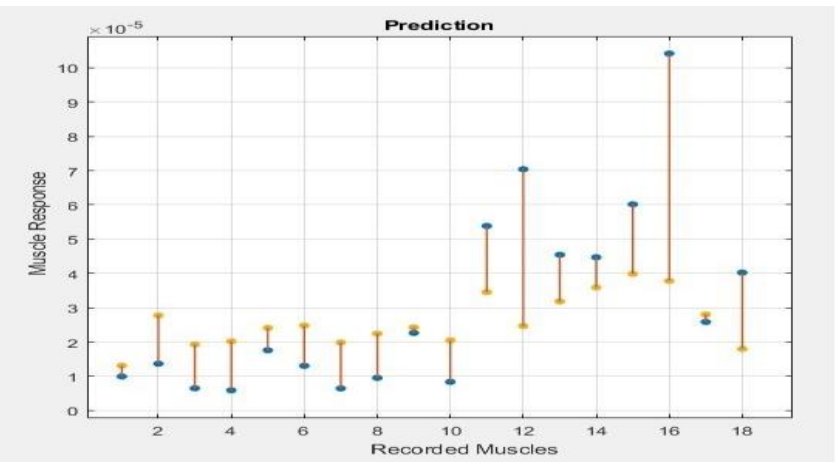

Fig. 4. Patient n2 (session 2), RMSE 7.3e-06, MSE 5.3e-11, MAE 5.4e-06.

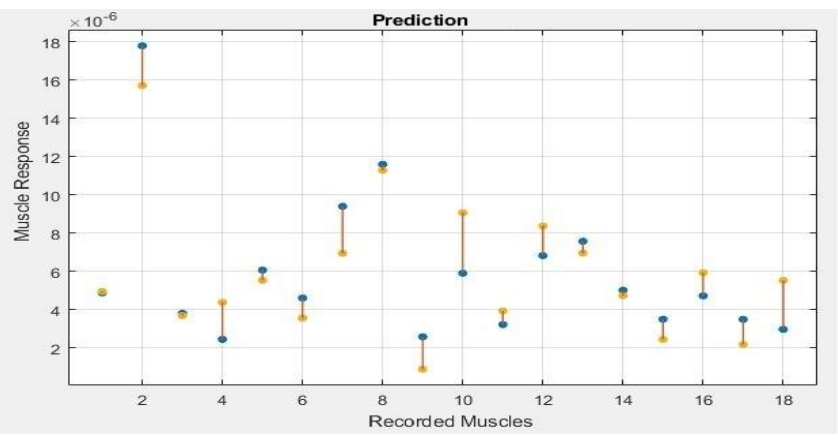

Fig. 5. Patient n3 (session 1), RMSE 6.6e-06, MSE 4.3e-11, MAE 5.1e-06.

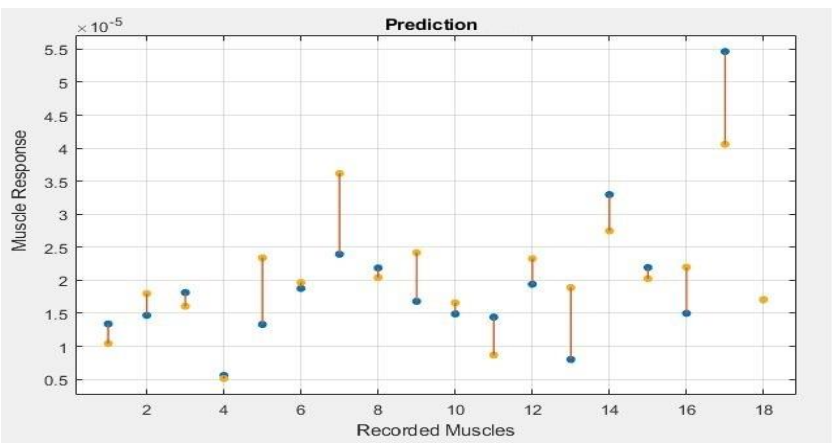

Fig. 6. Patient n3 (session 2), RMSE 1.5e-06, MSE 2.4e-12, MAE 1.3e-06.
From the above figures, we could observe that the trained model could predict, to some extent, the recorded data of the patients. For session 1, for instance, eight out of the nine of the stroke-affected muscles activities were well predicted for patients $1 \& 2$ ( 2 to 5 of the stroke-affected muscles were predicted for session-2 for the same patients). Patient 3, however, showed 5 out of 9 acceptable prediction of the affected-side muscles in both the sessions. Less predictions ability could be seen from the muscles in the non-affected side for all the three patients.

From the above results, although limited participants/data, we believe that the prediction level of the muscles on the stroke-affected is promising and open the challenge to continue the research on this direction. More data, in term of the number of subjects, as well as, number of recorded muscle activity history of each subject is essential to conclude better this study.

Regarding the poor ability of trained model to predict the muscle perforce of the non-affected side could be due to the high redundancy of healthy muscles activities compare to stroke affected muscle activities [7].

\section{CONCLUSION}

This paper presented a pilot study examines the ability to predict future muscle performance of post-stroke patients based on their current motor ability. The prediction model utilized SVMR method, trained by actual EMGs activities of stroke patients, and validated by their future muscle performance. A statistical validation and conclusion of this work would be possible by increasing the data-set size, which what we are planning for future direction.

\section{REFERENCES}

S. M. Mostafavi, J. I. Glasgow, S. P. Dukelow, S. H. Scott, and P. Mousavi, "Prediction of stroke-related diagnostic and prognostic measures using robot-based evaluation," in Rehabilitation Robotics (ICORR), 2013 IEEE International Conference on, 2013, pp. 1-6: IEEE.

G. M. Bani Musa, A. Al-Jumaily, F. Alnajjar, and S. Shimoda, "Analyze the Human Movements to Help CNS to Shape the Synergy using CNMF and Pattern Recognition," Procedia Computer Science, vol. 105, no. Supplement C, pp. 170-176, 2017/01/01 2017.

F. Alnajjar, M. Itkonen, V. Berenz, M. Tournier, C. Nagai, and S. Shimoda, "Sensory synergy as environmental input integration," Frontiers in Neuroscience, vol. 8, Jan. 2015.

F. Alnajjar, T. Wojtara, H. Kimura, and S. Shimoda, "Muscle synergy space: learning model to create an optimal muscle synergy," Frontiers in Computational Neuroscience, vol. 7, 2013.

Á. Costa, M. Itkonen, H. Yamasaki, F. Alnajjar, and S. Shimoda, "Importance of muscle selection for EMG signal analysis during upper limb rehabilitation of stroke patients," in Engineering in Medicine and Biology Society (EMBC), 2017 39th Annual International Conference of the IEEE, 2017, pp. 2510-2513: IEEE.

C. M. Stinear, W. D. Byblow, S. J. Ackerley, P. A. Barber, and M.-C. Smith, "Predicting recovery potential for individual stroke patients increases rehabilitation efficiency," Stroke, p. STROKEAHA. 116.015790, 2017.

F. Alnajjar, T. Wojtara, H. Kimura, and S. Shimoda, "Muscle synergy space: learning model to create an optimal muscle synergy," Frontiers in Computational Neuroscience, vol. 7, 2013 